
\title{
How does spaceflight affect the acquired immune system?
}

\author{
Taishin Akiyama $\mathbb{I D}^{1 凶}$, Kenta Horie ${ }^{1}$, Eiichi Hinoi $\mathbb{D}^{2,3}$, Manami Hiraiwa ${ }^{2,4}$, Akihisa Kato ${ }^{5,6,7}$, Yoichi Maekawa $\mathbb{I D}^{8,9}$, \\ Akihisa Takahashi $\left(D^{10}\right.$ and Satoshi Furukawa ${ }^{11}$
}

The impact of spaceflight on the immune system has been investigated extensively during spaceflight missions and in model experiments conducted on Earth. Data suggest that the spaceflight environment may affect the development of acquired immunity, and immune responses. Herein we summarize and discuss the influence of the spaceflight environment on acquired immunity. Bone marrow and the thymus, two major primary lymphoid organs, are evidently affected by gravitational change during spaceflight. Changes in the microenvironments of these organs impair lymphopoiesis, and thereby may indirectly impinge on acquired immunity. Acquired immune responses may also be disturbed by gravitational fluctuation, stressors, and space radiation both directly and in a stress hormone-dependent manner. These changes may affect acquired immune responses to pathogens, allergens, and tumors.

npj Microgravity (2020)6:14; https://doi.org/10.1038/s41526-020-0104-1

Astronauts experience hostile environmental changes during spaceflight, including microgravity, high doses of radiation, psychological stress induced by constraints and fears, and hypergravity during launching and landing. These factors reportedly affect many physiological systems in the body ${ }^{1-4}$. Several studies suggest that the immune system may be disturbed by spaceflight ${ }^{2-4}$. The immune system as a whole encompasses two main components, innate immunity and acquired immunity. Acquired immune responses are critical for inflammation, immunity against infection, and tumor immunity. In this review, we discuss how spaceflight environments may influence the development and functions of acquired immunity.

\section{ACQUIRED IMMUNE SYSTEM AND ITS DEVELOPMENT}

Lymphocytes play central roles in acquired immune responses. Almost all lymphocytes, including $T$ cells and B cells are originally derived from hematopoietic stem cells in the bone marrow ${ }^{5}$. While $B$ cells mature in the bone marrow, the progenitors of $T$ cells from bone marrow mature into $T$ cells in the thymus. Mature $T$ and $B$ cells migrate from primary lymphoid organs and are distributed to the various peripheral organs of the body ${ }^{6}$. Activation of the innate immune system by pathogen invasion triggers T-cell activation and differentiation via antigen presentation and the activities of various cytokines ${ }^{7}$. Activated effector $T$ cells further activate $B$ cells and macrophages during immune responses, and can also directly kill virus-infected cells $s^{8,9}$. After pathogens are eliminated, proportions of antigen-specific $T$ cells and B cells are converted into immunological memory cells that contribute to effective and long-lasting immunity against those pathogens ${ }^{10}$. Spaceflight may affect the initial development of lymphocytes, as well as their antigen-specific responses and the development of lymphocytic immunological memory, and thereby disrupt acquired immune responses ${ }^{11,12}$.

\section{IMPACT OF SPACEFLIGHT ON THE DEVELOPMENT OF ACQUIRED IMMUNITY}

Lymphoid organs are classified into primary lymphoid organs and secondary lymphoid organs, and both are required for the development and maintenance of efficient immune responses. Because the impact of spaceflight on lymphoid organ homeostasis is difficult to evaluate in humans, many spaceflight and groundbased model experiments have been conducted using rodents ${ }^{13-19}$.

The differentiation and maturation of B cells, myeloid cells, erythrocytes, hematopoietic stem cells (HSCs), and other progenitor cells occurs in the bone marrow, and bone is influenced by reduced gravity during spaceflight ${ }^{20-22}$. Therefore, spaceflight may change the bone marrow environment, thereby affecting immune systems. The bone marrow environment is composed of cells of hematopoietic origin (i.e., osteoclasts and macrophages), and non-hematopoietic stroma cells such as osteoblasts, fibroblasts, endothelial cells, and adipocytes ${ }^{23}$.

Mesenchymal stem cells (MSCs) are a source of osteoblasts, chondrocytes, and adipocytes in the bone tissue ${ }^{24}$ (Fig. 1a). They also regulate the functions of HSCs and other hematopoietic cells as niche cells ${ }^{23}$. Astronauts and patients who are bedridden longterm with little to no gravitational stimulation of their bones exhibit a sharp decrease in bone mass and immune function. This can be prevented via mechanical loading, such as that imparted during exercise $\mathrm{e}^{25}$. The role played by MSCs in dysfunction caused by microgravity environments is largely unclear, but associations between MSC characteristics and environmental gravitational changes have been reported (Fig. 1b) ${ }^{26,27}$. Microgravity inhibits osteogenic differentiation and increases the adipogenic

\footnotetext{
'Laboratory for Immune Homeostasis, RIKEN Center for Integrative Medical Sciences, Yokohama 230-0045, Japan. ${ }^{2}$ Laboratory of Pharmacology, Department of Bioactive

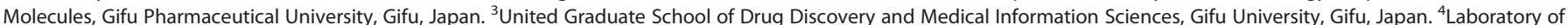
Molecular Pharmacology, Division of Pharmaceutical Sciences, Kanazawa University Graduate School, Kanazawa, Ishikawa, Japan. ${ }^{5}$ Division of Molecular Virology, Department of Microbiology and Immunology, The Institute of Medical Science, The University of Tokyo, Minato-ku, Tokyo 108-8639, Japan. ${ }^{6}$ The Institute of Medical Science, The University of Tokyo, Minato-ku, Tokyo 108-8639, Japan. ${ }^{7}$ Research Center for Asian Infectious Diseases, The Institute of Medical Science, The University of Tokyo, Minato-ku, Tokyo 108-8639,

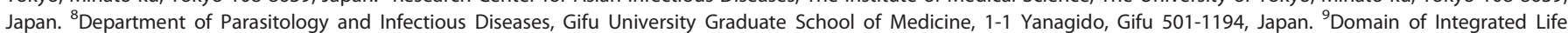

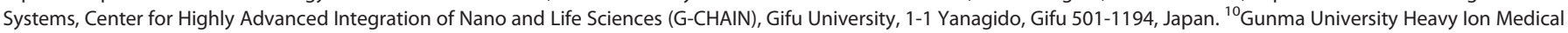
Center, Maebashi, Gunma 371-8511, Japan. ${ }^{11}$ Japan Aerospace Exploration Agency (JAXA), Tsukuba, Ibaraki 305-8505, Japan. ${ }^{凶}$ email: taishin.akiyama@riken.jp
} 

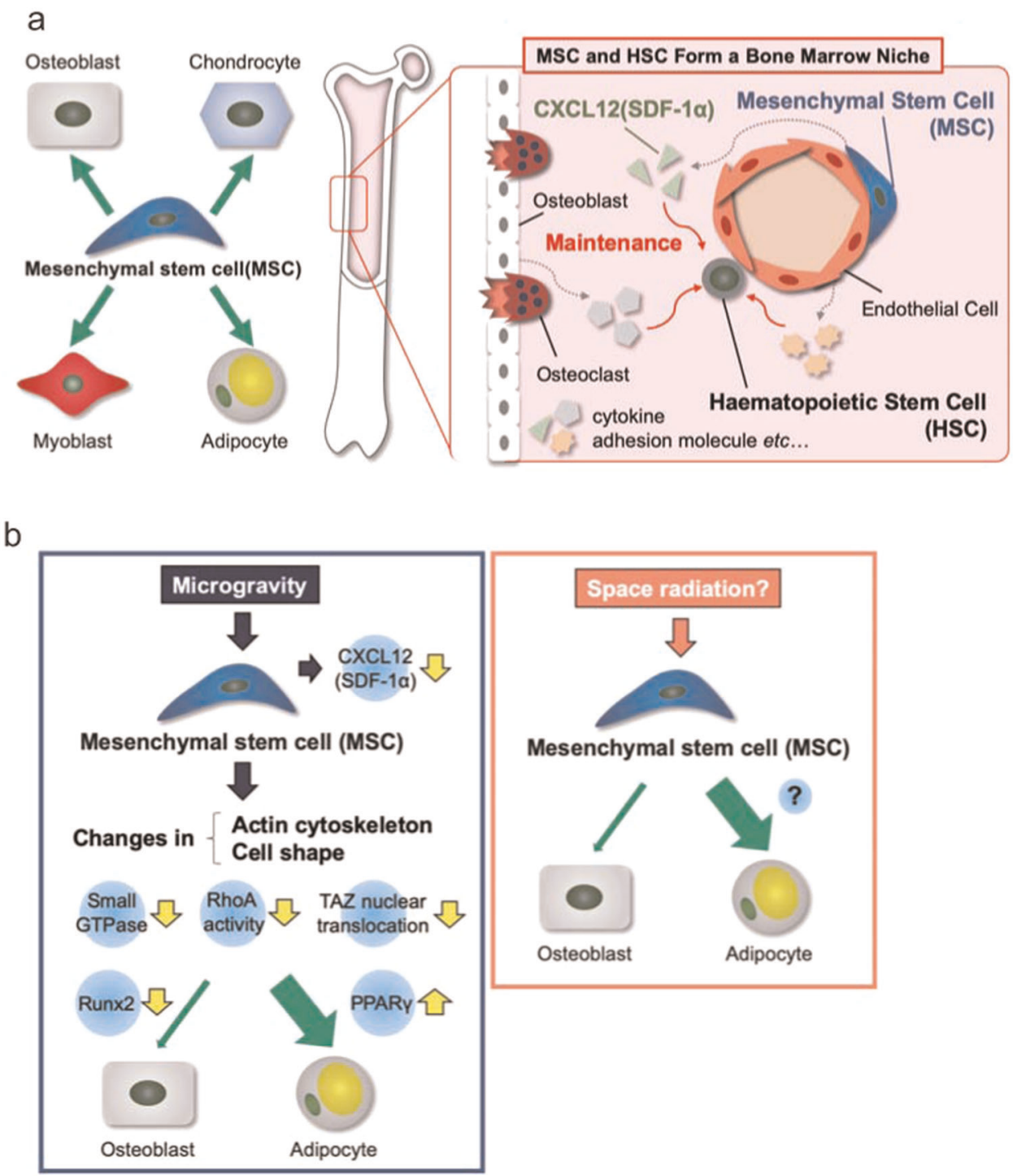

Fig. 1 Functions and differentiation of mesenchymal stem cells (MSCs) in bone marrow. : a Function and differentiation of MSCs in the homeostatic condition. $\mathbf{b}$ Alteration in differentiation of MSCs by microgravity and space radiation.

differentiation of MSCs, at least partly via the inhibition of Runx2 expression and the promotion of PPARy expression ${ }^{26}$. An imbalance in MSC lineage commitment to osteogenesis or adipogenesis is caused by changes in the actin cytoskeleton and cell shape via small GTPase, RhoA, and the transcriptional coactivator TAZ under microgravity conditions ${ }^{26,27}$.

MSCs and HSCs form a unique niche in the bone marrow microenvironment that regulates the stemness and function of stem cells and their lineage commitment by producing favorable factors $^{23}$. In human MSCs microgravity inhibits the expression of the chemokine CXCL12 (also known as SDF-1a), which is essential for maintaining HSC stemness and function as well as their retention in bone marrow, their quiescence, and their repopulating capacity ${ }^{28}$. This suggests that MSC dysfunction may be involved in the apparent decline in immune function and/or "space anemia" reported in astronauts via interaction with HSCs in the bone marrow microenvironment.

Regenerative medicine using MSCs has recently received significant attention. Fine tuning of the duration of exposure to simulated microgravity in addition to differentiation-inducing conditions can control MSC-lineage commitment to multiple functional cell types including osteoblasts (osteogenesis), chondrocytes (chondrogenesis), adipocytes (adipogenesis), endothelial cells (angiogenesis), and neurons (neurogenesis) ${ }^{29-31}$. Moreover, human MSCs cultured under simulated microgravity retain stem cell characteristics and the ability to differentiate into hyaline cartilage following transplantation ${ }^{32}$. A previous study in mice suggests that space radiation, which is a major environmental stressor in humans in space, may affect MSC differentiation into adipocytes ${ }^{33}$. In that study, MSCs in irradiated mice preferentially differentiated into adipocytes in the bone marrow. Elucidation of the mechanisms underlying the biological responses of MSCs to environmental stressors in humans in space may contribute to advances in regenerative medicine using MSCs, as well assisting the design of strategies to prevent bone loss in astronauts and patients with age-related and disuse-related osteoporosis.

A bone marrow environment is required for B-cell generation. Although B-cell frequency was reportedly not changed immediately after spaceflight, it was significantly reduced 1 week after landing ${ }^{34}$. Spaceflight did not affect the immunoglobulin repertoires of mice after short-time spaceflight ${ }^{35}$. Consistently, in a recent study B-cell homeostasis was maintained in astronauts during long-term spaceflight ${ }^{36}$. These data suggest that the 


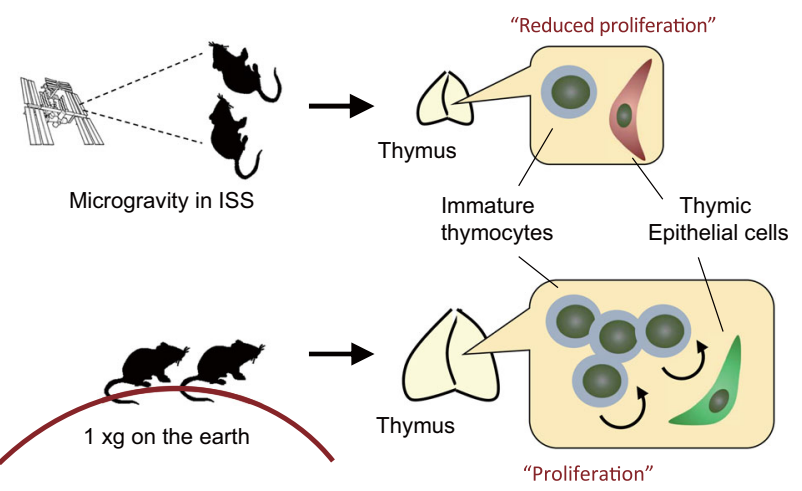

Fig. 2 Influence of spaceflight on the murine thymus. : In mice, spaceflight causes thymic atrophy that is most likely due to reduced cell proliferation.

influence of spaceflight on B-cell development and function may be limited.

The thymus is a primary lymphoid organ and generates almost all of the $T$ cells in the body ${ }^{37}$, and various physiological and psychological stressors can cause it to undergo atrophy ${ }^{38-40}$.

It is therefore not surprising that hostile environmental changes associated with spaceflight cause thymic atrophy (Fig. 2), and thereby may affect thymic functions. Impairment of thymic functions by spaceflight was suggested in a study that investigated human blood samples ${ }^{41}$. The recombination of T-cell antigen receptor genes during T-cell development in the thymus generates episomal circles containing the excised DNA (T-cell receptor excision circles; TRECs). As a result, naive T cells from the thymus can be detected via PCR analysis of TRECS ${ }^{42}$. TREC PCR analysis of blood samples from astronauts reportedly indicated that T-cell output from the thymus is reduced after spaceflight ${ }^{41}$, suggesting impairment of T-cell development in the thymus.

In addition to human sample analysis, the influence of spaceflight on the thymus has been investigated in rodents. Early studies performed during missions of $\sim 2$ weeks in space revealed that spaceflight caused an involution of the murine thymus ${ }^{16,43}$. In another relatively long-term spaceflight, there was also a reduction in murine thymic mass ${ }^{18}$. These data strongly suggest that spaceflight induces a thymic atrophy. Because mice would experience various kinds of environmental changes during spaceflight, however, it is difficult to determine what factors contribute to spaceflight-induced thymic atrophy.

The Japan Aerospace Exploration Agency (JAXA) recently developed an experimental platform in which mouse cages can be centrifuged to control the gravity experienced in the International Space Station (ISS) ${ }^{22,44}$. The thymi of mice exposed to $1 \mathrm{~g}$ in the ISS were recently analyzed ${ }^{45}$. Interestingly, exposure to experimentally imposed $1 \mathrm{~g}$ significantly alleviated spaceflightinduced thymic atrophy. Thus, altered gravity is presumably involved in thymic atrophy during spaceflight. Moreover, that result suggests that $1 \mathrm{~g}$ exposure may be an effective countermeasure against spaceflight-induced thymic atrophy. RNA sequencing analysis revealed down-regulation of genes regulating the $\mathrm{G} 2 / \mathrm{M}$ phase of the cell cycle in murine thymi after spaceflight, suggesting that impairment of thymic cell proliferation may induce thymic involution in mice during spaceflight (Fig. 2).

In addition to a reduction in cell cycle regulating genes, the thymic microenvironment is reportedly affected by spaceflight ${ }^{45}$. During T-cell differentiation in the thymus interactions between $\mathrm{T}$ cells and thymic epithelial cells (TECs) are critical ${ }^{46}$. TECs are largely divided into cortical TECs and medullary TECs (mTECs). TECs expressing keratin-5 (Krt5; Krt5 TECs) are normally localized in the medullar region of the thymus, and are therefore classified as mTECs. Interestingly, spaceflight caused mislocalization of Krt5 TECs in the cortex region, implying that spaceflight affected properties of
TECs. Given that TECs control the development and selection of thymocytes, it is possible that the disruptive effects of spaceflight on TECs indirectly caused a reduction in thymocytes. These changes in thymic gene expression and TEC localization associated with spaceflight are also alleviated by exposure to $1 \mathrm{~g}$ in the ISS, suggesting a critical role of altered gravity on these phenomena.

\section{IMPACT OF SPACEFLIGHT ENVIRONMENT ON IMMUNE CELL RESPONSES}

In addition to effects on immune development, spaceflight appears to lead to various other changes in immune responses. Samples from astronauts have been used to investigate the effects of spaceflight on the immune system ${ }^{12,47-55}$. In a seminal study in the early 1980s it was reported that neutrophils were elevated and eosinophils were reduced in the peripheral blood of astronauts who underwent spaceflight in the space shuttle ${ }^{56}$. Moreover, in vitro activation of T-cells prepared from astronauts was significantly reduced ${ }^{57,58}$. Further studies revealed that spaceflight affects various immune parameters such as the distribution of leukocytes ${ }^{12,47}$, granulocyte and monocyte function ${ }^{47,52}$, natural killer cell function ${ }^{53}$, and cytokine levels in plasma ${ }^{49,54}$ and in response to stimuli ${ }^{12,55}$

Given that many kinds of environmental changes and stressors are induced during spaceflight, each factor may directly disturb immune cell numbers and functions via individual mechanisms. In addition to direct effects, spaceflight-associated stressors increase levels of stress hormones (i.e., cortisol, dehydroepiandrosterone, epinephrine, and norepinephrine) via activation of hypothalamicpituitary-adrenal and sympathetic-adrenal-medullary axes ${ }^{59}$. Because these stress hormones are known to affect immune cells ${ }^{59}$, modulation of the immune system by spaceflight may be partly due to these humoral factors. Because of this complexity, the precise mechanisms underlying the effects of spaceflight remain to be determined.

Gravity change is one of the typical events that occurs during spaceflight, and it may influence immune systems by both direct mechanisms and indirect mechanisms via the induction of stress hormones. Spaceflight has high associated operating costs, therefore in addition to spaceflight experiments several groundbased models of spaceflight have been developed to investigate the effects of microgravity. Experimental methods incorporating low-gravity environments such as parabolic flight, a simulated microgravity environment such as a clinostat, long-term bed rest, and hindlimb suspension in experimental animals have been employed. It is necessary to carefully consider the extent to which these experimental methods actually reflect microgravity.

Tauber et al. ${ }^{60}$ reported that cell surface expression of CD3 and IL-2 receptor on non-activated T cells in human peripheral blood decreased just $20 \mathrm{~s}$ after low-gravity induction in a parabolic flight study. In addition, activated T cells placed in a two-dimensional clinostat as a simulated microgravity environment for $5 \mathrm{~min}$ exhibited reduced surface expression of CD3, reduced ZAP-70, and increased histone $\mathrm{H} 3$ acetylation. When these changes are induced in T cells in an actual microgravity environment, TCR signaling may be attenuated or modulated, markedly affecting immune responses. Importantly, because the influence of steroid hormones can be excluded in this system, the result indicated that the immune cell itself responds to gravitational fluctuation, suggesting the existence of a gravity sensor in immune cells.

Murine hindlimb suspension is a ground-based model primarily used to study the effects of microgravity on muscles and bones ${ }^{17}$. Stress during hindlimb unloading seems to be similar to spaceflight-induced stress with regard to causing activation of the sympathetic nervous system and the hypothalamic-pituitaryadrenal axis, thereby disrupting systemic immune responses ${ }^{17}$. Both CD4 T-cell and CD8 T-cell responses to mitogenic stimulation were reduced in mice that had undergone hindlimb suspension ${ }^{61}$. 
Notably however, hindlimb suspension caused a reduction in CD4 T cells and an increase in CD8 T cells in the spleen ${ }^{61}$. The reason for this apparent inconsistency remains unknown, but one possibility is that CD4 T cells may be more sensitive to stress induced by hindlimb suspension than CD8 $\mathrm{T}$ cells, and the increase in CD8 T cells may be due to homeostatic proliferation that is induced by reduction in the overall T-cell population. Notably, given that hindlimb suspension is not a perfect model for studying systemic in vivo effects, further research incorporating spaceflight experiments will be necessary to facilitate definitive conclusions.

\section{EFFECTS OF SPACEFLIGHT ON IMMUNITY AND THE ONSET OF DISEASE}

There have been no reports of astronauts developing serious infectious diseases during spaceflight or after returning, but it is currently unclear whether long-term exposure to continuous microgravity can promote infectious diseases. Because astronauts who are selected for space travel are both mentally and physically healthy, it is highly probable that the modulation of the immune system due to gravitational fluctuations may be affected by their robustness or homeostatic mechanisms. Alternatively, an immune system that has been modulated by a period of stay may still have a sufficient margin until the onset of serious infection. Notably, however, in a previous study astronauts who stayed in a space shuttle for a short period (12-16 days) before, during, and after the flight exhibited reactivation of latent viral infections 49 .

Approximately $70-95 \%$ of the global population is estimated to be infected with at least one of the nine known human herpes viruses $(\mathrm{HHVs})^{62}$. The vast majority of $\mathrm{HHV}$ infections are considered clinically asymptomatic phases of infection referred to as latency ${ }^{62}$. However, space is expected to be a high-risk environment with regard to disturbing the symbiotic relationship between humans and latent infectious viruses. Reactivation and/ or shedding of latent HHVs such as Varicella-zoster virus (VZV), EBV, and human cytomegalovirus has been reported in astronauts during the Russian Soyuz and ISS missions ${ }^{49,59,63}$. Furthermore, the National Aeronautics and Space Administration (NASA) reported that (i) VZV was detected in the saliva of $\sim 50 \%$ of astronauts on flight and approximately $60 \%$ of astronauts during the flight phase of the ISS; and (ii) astronauts occasionally experienced cold sores, which are usually caused by herpes simplex virus type-1 (HSV-1), although the occurrence of HSV-1 in saliva was rare (https:// humanresearchroadmap.nasa.gov/Evidence/reports/Host_Micro.pdf).

HSV-1 and VZV are neurotropic human alpha herpes viruses. After epithelial entry viral particles enter a plexus of sensory neurons, gaining access to neuronal axons. Once these viruses reach the neuronal body, effector memory CD8 and CD4 lymphocytes establish residence, and the viral genomes persist in latency in the trigeminal ganglion or dorsal root ganglion ${ }^{64,65}$. The immune response plays an important role in the ganglia during infection with HSV-1 ${ }^{66}$. HSV-1 latency in sensory ganglia is characterized by persistent CD8 T-cell infiltration ${ }^{67}$, and the recovery of infectious viral particles from ex vivo explants of latently infected murine trigeminal ganglions following ocular infection is completely inhibited in the presence of CD8 T cells ${ }^{68}$. Furthermore, depletion of CD8 T cells from latently infected neuronal nodes can promote HSV-1 reactivation ${ }^{68}$. Notably, Knickelbein et al. ${ }^{69}$ reported that granzyme $B$ release by CD8 T cells inhibited HSV-1 reactivation by degrading the essential viral transcription factor HSV-1 infected-cell polypeptide 4 (Fig. 3). From these findings, it is reasonable to conclude that space-induced impairment of adaptive immunity may be implicated in establishing HSV-1 and/or VZV latency.

Vaccines are an effective strategy for preventing viral diseases. No approved HSV-1 vaccine exists but a live attenuated VZV derived from $\mathrm{Oka}^{70}$ and a subunit vaccine containing VZV glycoprotein E and the AS01B adjuvant system ${ }^{71}$ are available to prevent both chickenpox and herpes zoster. The development of vaccines for other HHVs is desirable for administration to humans living in space. In the future, space travelers may utilize spacespecific vaccines. Although the individual effects of the extreme stressors experienced in space such as gravity changes or radiation on the symbiotic relationship between humans and HHVs have not been elucidated, changes in the regulation of the host immune system are considered an important factor.

Because experimental infection in a spaceship would be problematic, some model ground-based experiments have been performed to investigate the influence of spaceflight-type environments on infectious immunity. Weisman et al. ${ }^{72}$ reported a synergistic effect of hindlimb suspension and radiation on the control of bacterial infection. The combination of hindlimb suspension and proton irradiation significantly increased the lethality of bacterial infection in mice after intraperitoneal inoculation with Pseudomonas aeruginosa above that of either treatment alone. A similar phenomenon was observed with the combination of hindlimb suspension and gamma irradiation. In the combined group there was almost no increase in granulocytes in blood against the infection. Total serum corticosterone was highest in the combined group. Zhou et al. ${ }^{71}$ reported similar effects of a different combined treatment (complex stress) on the control of intestinal microbiota. Serum lipopolysaccharides (LPS) increased $6 \mathrm{~h}$ after proton beam irradiation or hindlimb suspension. Because LPS elevations in serum may be derived from Gramnegative bacteria in the intestinal tract, these treatments resulted in a breakdown in the integrity of the intestinal epithelium. Moreover, the combined treatment of hindlimb suspension and irradiation exerted synergistic effects on LPS elevation. LPS elevation was transient after a single treatment, but it continued for at least 4 days after irradiation in the combined treatment group. In conjunction with the increase in serum LPS concentrations, serum LPS binding protein and soluble CD14 also increased. Furthermore, the inflammatory cytokines IL-6, tumor necrosis factor- $a$, and IFN- $a$ were elevated. So-called LPS tolerance is induced by continuous stimulation with LPS. Spleen cells from mice that underwent 13 days of spaceflight reportedly exhibited

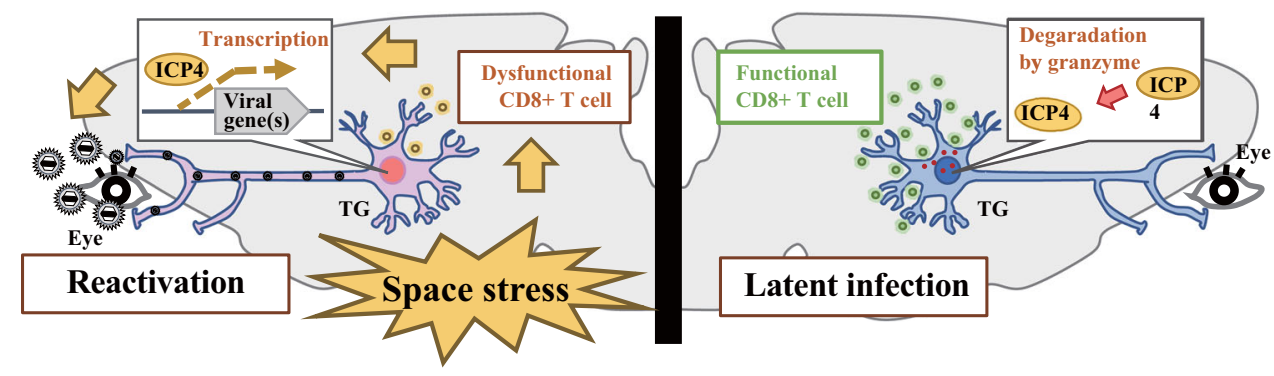

Fig. 3 Reactivation of latent virus during spaceflight. Space stressors may cause dysfunction of CD8 T cells that in turns induces reactivation of HSV-1. 
reduced tumor necrosis factor-a production and low blastogenesis in response to LPS stimulation ${ }^{15}$. LPS tolerance may occur due to exposure to LPS, but reported spleen cell responses to mitogenic stimuli such as LPS vary between studies, thus further investigation is required in this regard.

In addition to compromised immune responses, spaceflight may affect the onset and progression of immunological conditions such as allergy and autoimmunity. Some astronauts experience allergy-like symptoms during spaceflight ${ }^{73}$. Moreover, skin rashes and hypersensitivity frequently occur in astronauts ${ }^{74}$. These reports suggest dysregulation of immune and inflammatory responses by spaceflight environments. The mechanisms underlying this dysregulation remain unclear. Various stressors can be risk factors for such symptoms ${ }^{75}$, thus it is possible that psychological stress during spaceflight indirectly contributes to their onset and/or progression. To date there is no clear evidence that spaceflight is associated risk of developing autoimmunity.

\section{SPACE-INDUCED IMPAIRMENT OF IMMUNITY: WILL IT INCREASE THE RISK OF CANCER?}

The acquired immune system plays a major role in preventing tumor growth and metastasis, suggesting that immune system aberrations caused by stressors associated with space travel should be considered when estimating the risk of cancer-related mortality. Interestingly, studies to date have not detected significant differences in cancer mortality between astronauts and non-astronauts ${ }^{76-79}$ or between deep spaceflight astronauts and non-flight astronauts ${ }^{80}$. For reasons that have not been elucidated, astronauts do reportedly exhibit significantly higher rates of accidental death than non-astronauts ${ }^{76-80}$. Notably, it may be too early to draw definitive conclusions on health risks in astronauts, due to the small numbers surveyed and the fact that they tend to spend relatively short periods of time in space. Similarly, due to a lack of relevant information we cannot predict how travelling to the Moon or Mars, or long periods of time in deep space may affect health, including the risk of cancer.

Over the past 20 years risk assessments associated with space radiation have mainly focused on cancer. Space radiation can certainly damage DNA and induce genetic changes that may lead to cancer ${ }^{81,82}$. For example, damage such as double-strand break tracks have been detected in the nuclei of cells exposed to space radiation $^{82}$, and increased frequencies of chromosomal aberrations have been detected in lymphocytes from astronauts during and after spaceflight. Many cancers may not emerge until years after the initial contributory genetic insults, which is relevant to a Mars mission with a potential duration of approximately 3 years because space-induced cancer may be detected after, but not during, spaceflight. Of course, astronauts can receive standard cancer treatment after spaceflight.

In addition to cancer risk assessment, NASA has recently increased its focus on space-induced central nervous system and cardiovascular diseases. The galactic cosmic ray simulator developed by NASA ${ }^{83}$ enables rapid switching between various highenergy ion sources, more closely simulating the radiation exposure experienced in space. The NASA space radiation program has encouraged researchers to investigate exposure to simulated solar particle events and galactic cosmic rays as part of their space radiation research. In the past, space-induced cancer risk assessment has been based on radiation quality and quantity, but the added risk of exposure to space radiation under microgravity conditions is unknown. In the near future astronauts and civilians potentially harboring undetectable micro-cancers may undertake long-term stays in space, which poses a substantial problem.

We previously reported that, in studies using our simulated microgravity-irradiation system ${ }^{84,85}$, simultaneous exposure of human fibroblasts to simulated microgravity and radiation had greater effects than radiation exposure alone on the expression of cell cycle-related genes associated with genomic instability ${ }^{86}$, and on the frequency of chromosomal aberrations ${ }^{87}$. It has also been reported that in a murine hindlimb suspension model simulating microgravity, hindlimb suspension significantly increased spindle cancer tumor growth and caused splenic atrophy in wild-type mice ${ }^{88}$. In that same study, tumor growth in severe combined immunodeficient (so-called "SCID") mice did not differ significantly from tumor growth in wild-type controls. We also recently reported that hindlimb suspension significantly increased osteosarcoma tumor growth and metastasis to the lung, and caused greater thymic atrophy compared with mice in constant orthostatic suspension or unmanipulated mice under standard housing conditions $^{89}$. Notably, temporary loading prevented these adverse effects ${ }^{89}$. Future studies should investigate irradiation-induced carcinogenesis in the hindlimb suspension model and other mouse models.

Although hindlimb suspension has been utilized in the study of adverse consequences of spaceflight, it is not a perfect model of microgravity. In addition, the mechanisms of systemic responses in the hindlimb suspension model remain unclear. Therefore, it is necessary to verify the results of hindlimb suspension studies in space-based experiments. The new mouse habitat cage units developed by JAXA enable mice to be exposed to simulated microgravity and partial gravity conditions ${ }^{22}$. If this device can be used in deep space, as the Deep Space Gateway habitat can, the resulting experiments will be of vital importance in clarifying the cancer risks associated with changes in gravity, in addition to reduced immunity and radiation-induced genome instability. We hope that such experiments will increase the accuracy of cancer risk assessments related to space travel.

\section{PROBIOTIC AND PREBIOTIC INTAKE AS POTENTIAL IMMUNE COUNTERMEASURES}

Crucian et al. ${ }^{11,90}$ and Makedonas et al. ${ }^{11,90}$ reviewed a range of potential immune countermeasures including exercise, stress management, medications, and nutrition/supplementation. Below, we specifically focus on probiotic and prebiotic intake as possible countermeasures. Probiotics are currently defined as "live strains of strictly selected microorganisms which, when administered in adequate amounts, confer a health benefit on the host ${ }^{\prime 91}$. The effects of various kinds of probiotic supplementation on the health status of athletes have been reported ${ }^{92,93}$. An ongoing study by the JAXA is investigating the effects of probiotic (Lactobacillus casei Shirota) supplementation on intestinal microbiota and immune function in astronauts. The results of those studies will yield information on possible countermeasures.

The definition of a prebiotic was updated as a substrate that is selectively utilized by host microorganisms conferring a health benefit ${ }^{94}$. Fructooligosaccharides (FOS) are considered prebiotics. FOS promote the bifidobacteria growth ${ }^{95}$. In addition, FOS can indirectly and directly influence immune function through increased short chain fatty acid (SCFA) production, which modifies the interleukin production and natural killer cell activity ${ }^{96}$, and modification of the immune system via the gut-associated lymphoid tissue ${ }^{97}$. In fact, another ongoing study by JAXA is examining the effects of prebiotic (FOS) supplementation on host immunity using human and mouse specimen ${ }^{44}$. The results will give us more insights into countermeasure strategy. Furthermore, even synbiotics, which is a combination of synergistically acting probiotics and prebiotics introduced by Gibson and Roberfroid in $1995^{98}$, would be a candidate for effective countermeasures for future human exploration missions. In a prospective, randomized, double blind, placebo-controlled study on Earth, it was reported that intake of synbiotic formulations containing 3 to 5 strains of Lactobacillus plantarum, Lactobacillus rhamnosus, and Bifidobacterium lactis, lactoferrin and prebiotics (FOS or galactooligosaccharides) reduced the incidence and severity of URTIs during the cold 
season $^{99}$. Further studies using ground analogs, for example international isolation projects and Antarctic missions, would enable to validate the countermeasure candidates.

\section{DATA AVAILABILITY}

Data available on request from the authors.

Received: 21 December 2019; Accepted: 17 April 2020; Published online: 07 May 2020

\section{REFERENCES}

1. Demontis, G. C. et al. Human pathophysiological adaptations to the space environment. Front Physiol. 8, 547 (2017).

2. Crucian, B. et al. Terrestrial stress analogs for spaceflight associated immune system dysregulation. Brain Behav. Immun. 39, 23-32 (2014).

3. Garrett-Bakelman, F. E. The NASA Twins Study: A multidimensional analysis of a year-long human spaceflight. Science 364, eaau8650 (2019).

4. Gueguinou, N. et al. Could spaceflight-associated immune system weakening preclude the expansion of human presence beyond Earth's orbit? J. Leukoc. Biol. 86, 1027-1038 (2009).

5. Weissman, I. L. Stem cells: units of development, units of regeneration, and units in evolution. Cell 100, 157-168 (2000).

6. Boehm, T., Hess, I. \& Swann, J. B. Evolution of lymphoid tissues. Trends Immunol. 33, 315-321 (2012).

7. Akira, S., Uematsu, S. \& Takeuchi, O. Pathogen recognition and innate immunity. Cell 124, 783-801 (2006).

8. Castellino, F. \& Germain, R. N. Cooperation between CD4+ and CD8+ T cells: when, where, and how. Annu Rev. Immunol. 24, 519-540 (2006).

9. Rouse, B. T. \& Sehrawat, S. Immunity and immunopathology to viruses: what decides the outcome? Nat. Rev. Immunol. 10, 514-526 (2010).

10. Farber, D. L., Netea, M. G., Radbruch, A., Rajewsky, K. \& Zinkernagel, R. M. Immunological memory: lessons from the past and a look to the future. Nat. Rev. Immunol. 16, 124-128 (2016).

11. Crucian, B. E. et al. Immune system dysregulation during spaceflight: potential countermeasures for deep space exploration missions. Front. Immunol. 9, 1437 (2018).

12. Crucian, B. et al. Alterations in adaptive immunity persist during long-duration spaceflight. NPJ Microgravity 1, 15013 (2015).

13. Sonnenfeld, G. Use of animal models for space flight physiology studies, with special focus on the immune system. Gravit. Space Biol. Bull. 18, 31-35 (2005).

14. Horie, K. et al. Down-regulation of GATA1-dependent erythrocyte-related genes in the spleens of mice exposed to a space travel. Sci. Rep. 9, 7654 (2019).

15. Baqai, F. P. et al. Effects of spaceflight on innate immune function and antioxidant gene expression. J. Appl Physiol. 106, 1935-1942 (2009).

16. Gridley, D. S. et al. Changes in mouse thymus and spleen after return from the STS-135 mission in space. PLOS ONE 8, e75097 (2013).

17. Globus, R. K. \& Morey-Holton, E. Hindlimb unloading: rodent analog for microgravity. J. Appl Physiol. (1985) 120, 1196-1206 (2016).

18. Novoselova, E. G. et al. Changes in immune cell signalling, apoptosis and stress response functions in mice returned from the BION-M1 mission in space. Immunobiology 220, 500-509 (2015).

19. Pecaut, M. J. et al. Genetic models in applied physiology: selected contribution: effects of spaceflight on immunity in the C57BL/6 mouse. I. Immune population distributions. J. Appl Physiol. 94, 2085-2094 (2003).

20. Vico, L. \& Hargens, A. Skeletal changes during and after spaceflight. Nat. Rev. Rheumatol. 14, 229-245 (2018).

21. Grimm, D. et al. The impact of microgravity on bone in humans. Bone $\mathbf{8 7}, \mathbf{4 4 - 5 6}$ (2016).

22. Shiba, D. et al. Development of new experimental platform 'MARS'-Multiple Artificial-gravity Research System-to elucidate the impacts of micro/partial gravity on mice. Sci. Rep. 7, 10837 (2017).

23. Mendez-Ferrer, S. et al. Mesenchymal and haematopoietic stem cells form a unique bone marrow niche. Nature 466, 829-834 (2010).

24. Pittenger, M. F. et al. Multilineage potential of adult human mesenchymal stem cells. Science 284, 143-147 (1999).

25. Collet, P. et al. Effects of 1- and 6-month spaceflight on bone mass and biochemistry in two humans. Bone 20, 547-551 (1997).

26. Meyers, V. E., Zayzafoon, M., Gonda, S. R., Gathings, W. E. \& McDonald, J. M. Modeled microgravity disrupts collagen I/integrin signaling during osteoblastic differentiation of human mesenchymal stem cells. J. Cell Biochem. 93, 697-707 (2004).
27. Chen, Z., Luo, Q., Lin, C., Kuang, D. \& Song, G. Simulated microgravity inhibits osteogenic differentiation of mesenchymal stem cells via depolymerizing F-actin to impede TAZ nuclear translocation. Sci. Rep. 6, 30322 (2016).

28. Gershovich, P. M., Gershovich, lu,G. \& Buravkova, L. B. [The effects of simulated microgravity on the pattern of gene expression in human bone marrow mesenchymal stem cells under osteogenic differentiation]. Fiziol. Cheloveka 39, 105-111 (2013).

29. Xue, L., Li, Y. \& Chen, J. Duration of simulated microgravity affects the differentiation of mesenchymal stem cells. Mol. Med Rep. 15, 3011-3018 (2017).

30. Zhang, X. et al. Model microgravity enhances endothelium differentiation of mesenchymal stem cells. Naturwissenschaften 100, 125-133 (2013).

31. Chen, J. et al. The simulated microgravity enhances the differentiation of mesenchymal stem cells into neurons. Neurosci. Lett. 505, 171-175 (2011).

32. Yuge, L. et al. Microgravity potentiates stem cell proliferation while sustaining the capability of differentiation. Stem Cells Dev. 15, 921-929 (2006).

33. Mizoguchi, T. et al. Osterix marks distinct waves of primitive and definitive stromal progenitors during bone marrow development. Dev. Cell 29, 340-349 (2014).

34. Tascher, G. et al. Analysis of femurs from mice embarked on board BION-M1 biosatellite reveals a decrease in immune cell development, including $B$ cells, after 1 wk of recovery on Earth. FASEB J. 33, 3772-3783 (2019).

35. Ward, C. et al. Effects of spaceflight on the immunoglobulin repertoire of unimmunized C57BL/6 mice. Life Sci. Space Res. 16, 63-75 (2018).

36. Spielmann, G. et al. B cell homeostasis is maintained during long-duration spaceflight. J. Appl Physiol. (1985) 126, 469-476 (2019).

37. Rothenberg, E. V., Moore, J. E. \& Yui, M. A. Launching the T-cell-lineage developmental programme. Nat. Rev. Immunol. 8, 9-21 (2008).

38. Majumdar, S. \& Nandi, D. Thymic atrophy: experimental studies and therapeutic interventions. Scand. J. Immunol. 87, 4-14 (2018).

39. Nunes-Alves, C., Nobrega, C., Behar, S. M. \& Correia-Neves, M. Tolerance has its limits: how the thymus copes with infection. Trends Immunol. 34, 502-510 (2013).

40. Dooley, J. \& Liston, A. Molecular control over thymic involution: from cytokines and microRNA to aging and adipose tissue. Eur. J. Immunol. 42, 1073-1079 (2012).

41. Benjamin, C. L. et al. Decreases in thymopoiesis of astronauts returning from space flight. JCl Insight 1, e88787 (2016).

42. Ribeiro, R. M. \& Perelson, A. S. Determining thymic output quantitatively: using models to interpret experimental T-cell receptor excision circle (TREC) data. Immunol. Rev. 216, 21-34 (2007).

43. Gridley, D. S. et al. Genetic models in applied physiology: selected contribution: effects of spaceflight on immunity in the C57BL/6 mouse. II. Activation, cytokines, erythrocytes, and platelets. J. Appl Physiol. 94, 2095-2103 (2003).

44. Matsuda, C. et al. Dietary intervention of mice using an improved Multiple Artificial-gravity Research System (MARS) under artificial 1 g. NPJ Microgravity 5, 16 (2019).

45. Horie, K. et al. Impact of spaceflight on the murine thymus and mitigation by exposure to artificial gravity during spaceflight. Sci. Rep. 9, 19866 (2019).

46. Abramson, J. \& Anderson, G. Thymic Epithelial Cells. Annu Rev. Immunol. 35, 85-118 (2017).

47. Stowe, R. P. et al. Leukocyte subsets and neutrophil function after short-term spaceflight. J. Leukoc. Biol. 65, 179-186 (1999).

48. Crucian, B. et al. Immune system dysregulation occurs during short duration spaceflight on board the space shuttle. J. Clin. Immunol. 33, 456-465 (2013).

49. Mehta, S. K. et al. Reactivation of latent viruses is associated with increased plasma cytokines in astronauts. Cytokine 61, 205-209 (2013).

50. Barrila, J. et al. Spaceflight modulates gene expression in the whole blood of astronauts. NPJ Microgravity 2, 16039 (2016).

51. Kunz, H. et al. Alterations in hematologic indices during long-duration spaceflight. BMC Hematol. 17, 12 (2017).

52. Kaur, I., Simons, E. R., Castro, V. A., Mark Ott, C. \& Pierson, D. L. Changes in neutrophil functions in astronauts. Brain Behav. Immun. 18, 443-450 (2004).

53. Konstantinova, I. V. et al. Natural killer cells after ALTAIR mission. Acta Astronaut. 36, 713-718 (1995).

54. Crucian, B. E. et al. Plasma cytokine concentrations indicate that in vivo hormonal regulation of immunity is altered during long-duration spaceflight. J. Interferon Cytokine Res. 34, 778-786 (2014).

55. Crucian, B. E., Cubbage, M. L. \& Sams, C. F. Altered cytokine production by specific human peripheral blood cell subsets immediately following space flight. $J$. Interferon Cytokine Res. 20, 547-556 (2000).

56. Taylor, G. R. \& Dardano, J. R. Human cellular immune responsiveness following space flight. Aviat. Space Environ. Med. 54, S55-S59 (1983).

57. Cogoli, A. \& Tschopp, A. Lymphocyte reactivity during spaceflight. Immunol. Today 6, 1-4 (1985).

58. Cogoli, A., Tschopp, A. \& Fuchs-Bislin, P. Cell sensitivity to gravity. Science 225, 228-230 (1984). 
59. Rooney, B. V., Crucian, B. E., Pierson, D. L., Laudenslager, M. L. \& Mehta, S. K. Herpes virus reactivation in astronauts during spaceflight and its application on earth. Front Microbiol 10, 16 (2019).

60. Tauber, S. et al. Signal transduction in primary human T lymphocytes in altered gravity during parabolic flight and clinostat experiments. Cell Physiol. Biochem $\mathbf{3 5}$ 1034-1051 (2015).

61. Gaignier, F. et al. Three weeks of murine hindlimb unloading induces shifts from $B$ to $T$ and from th to tc splenic lymphocytes in absence of stress and differentially reduces cell-specific mitogenic responses. PLoS ONE 9, e92664 (2014).

62. Roizman, B, Kimberlin, D. W. \& Whitley, R. J. Herpes simplex Viruses. 6th edn., ((Lippincott-Williams \& Wilkins: Philadelphia, PA, 2013.

63. Mehta, S. K. et al. Latent virus reactivation in astronauts on the international space station. NPJ Microgravity 3, 11 (2017).

64. Shin, H. \& Iwasaki, A. Tissue-resident memory T cells. Immunol. Rev. 255, 165-181 (2013)

65. Kennedy, P. G., Rovnak, J., Badani, H. \& Cohrs, R. J. A comparison of herpes simplex virus type 1 and varicella-zoster virus latency and reactivation. J. Gen. Virol. 96, 1581-1602 (2015)

66. Khanna, K. M., Lepisto, A. J., Decman, V. \& Hendricks, R. L. Immune control of herpes simplex virus during latency. Curr. Opin. Immunol. 16, 463-469 (2004).

67. Verjans, G. M. et al. Selective retention of herpes simplex virus-specific T cells in latently infected human trigeminal ganglia. Proc. Natl Acad. Sci. USA 104, 3496-3501 (2007).

68. Khanna, K. M., Bonneau, R. H., Kinchington, P. R. \& Hendricks, R. L. Herpes simplex virus-specific memory CD8 $+\mathrm{T}$ cells are selectively activated and retained in latently infected sensory ganglia. Immunity 18, 593-603 (2003).

69. Knickelbein, J. E. et al. Noncytotoxic lytic granule-mediated CD8+ T cell inhibition of HSV-1 reactivation from neuronal latency. Science 322, 268-271 (2008).

70. Asano, Y. et al. Experience and reason: twenty-year follow-up of protective immunity of the Oka strain live varicella vaccine. Pediatrics 94, 524-526 (1994).

71. Zhou, Y. et al. Effect of solar particle event radiation and hindlimb suspension on gastrointestinal tract bacterial translocation and immune activation. PLOS ONE 7 , e44329 (2012).

72. Li, M. et al. Hindlimb suspension and SPE-like radiation impairs clearance of bacterial infections. PLoS ONE 9, e85665 (2014).

73. Crucian, B. et al. Incidence of clinical symptoms during long-duration orbital spaceflight. Int J. Gen. Med 9, 383-391 (2016).

74. Crucian, B. et al. A case of persistent skin rash and rhinitis with immune system dysregulation onboard the International Space Station. J. Allergy Clin. Immunol. Pr. 4, 759-762 e758 (2016)

75. Montoro, J. et al. Stress and allergy. J. Investig. Allergol. Clin. Immunol. 19(Suppl 1), 40-47 (2009).

76. Peterson, L. E., Pepper, L. J., Hamm, P. B. \& Gilbert, S. L. Longitudinal study of astronaut health: mortality in the years 1959-1991. Radiat. Res. 133, 257-264 (1993).

77. Hamm, P. B., Billica, R. D., Johnson, G. S., Wear, M. L. \& Pool, S. L. Risk of cancer mortality among the Longitudinal Study of Astronaut Health (LSAH) participants. Aviat. Space Environ. Med. 69, 142-144 (1998).

78. Hamm, P. B., Nicogossian, A. E., Pool, S. L., Wear, M. L. \& Billica, R. D. Design and current status of the longitudinal study of astronaut health. Aviat. Space Environ. Med. 71, 564-570 (2000).

79. Reynolds, R. J. \& Day, S. M. Mortality among U.S. astronauts: 1980-2009. Aviat Space Environ. Med. 81, 1024-1027 (2010).

80. Delp, M. D., Charvat, J. M., Limoli, C. L., Globus, R. K. \& Ghosh, P. Apollo Lunar Astronauts Show Higher Cardiovascular Disease Mortality: Possible Deep Space Radiation Effects on the Vascular Endothelium. Sci. Rep. 6, 29901 (2016).

81. Ohnishi, T. et al. Detection of DNA damage induced by space radiation in Mir and space shuttle. J. Radiat. Res. 43(Suppl), S133-S136 (2002).

82. Ohnishi, T. et al. Detection of space radiation-induced double strand breaks as a track in cell nucleus. Biochem. Biophys. Res. Commun. 390, 485-488 (2009).

83. La Tessa, C., Sivertz, M., Chiang, I. H., Lowenstein, D. \& Rusek, A. Overview of the NASA space radiation laboratory. Life Sci. Space Res. 11, 18-23 (2016).

84. Ikeda, $H$. et al. A new system for three-dimensional clinostat synchronized $X-$ irradiation with a high-speed shutter for space radiation research. Biol. Sci. Space 30, 8-16 (2016).

85. Ikeda, $\mathrm{H}$. et al. Development and performance evaluation of a three-dimensional clinostat synchronized heavy-ion irradiation system. Life Sci. Space Res. 12, 51-60 (2017).

86. Ikeda, H. et al. Expression profile of cell cycle-related genes in human fibroblasts exposed simultaneously to radiation and simulated microgravity. Int. J. Mol. Sci. 20, 4791 (2019).

87. Hada, M. et al. Increased chromosome aberrations in cells exposed simultaneously to simulated microgravity and radiation. Int. J. Mol. Sci. 20, 43 (2019).

88. Lee, E. H., Ding, W., Kulkarni, A. D. \& Granstein, R. D. Tumor growth and immune function in mice during hind-limb unloading. Aviat. Space Environ. Med. 76, 536-540 (2005).
89. Takahashi, A. et al. Temporary Loading Prevents Cancer Progression and Immune Organ Atrophy Induced by Hind-Limb Unloading in Mice. Int. J. Mol. Sci. 19, 3959 (2018).

90. Makedonas, G. et al. Specific Immunologic Countermeasure Protocol for DeepSpace Exploration Missions. Front. Immunol. 10, 2407 (2019).

91. Hill, C. et al. Expert consensus document. The International Scientific Association for Probiotics and Prebiotics consensus statement on the scope and appropriate use of the term probiotic. Nat. Rev. Gastroenterol. Hepatol. 11, 506-514 (2014).

92. West, N. P. et al. Probiotic supplementation for respiratory and gastrointestinal illness symptoms in healthy physically active individuals. Clin. Nutr. 33, 581-587 (2014).

93. Gleeson, M., Bishop, N. C., Oliveira, M. \& Tauler, P. Daily probiotic's (Lactobacillus casei Shirota) reduction of infection incidence in athletes. Int J. Sport Nutr. Exerc Metab. 21, 55-64 (2011).

94. Gibson, G. R. et al. Expert consensus document: The International Scientific Association for Probiotics and Prebiotics (ISAPP) consensus statement on the definition and scope of prebiotics. Nat. Rev. Gastroenterol. Hepatol. 14, 491-502 (2017).

95. Rolim, P. M. Development of prebiotic food products and health benefits. Food Sci. Technol. 35, 3-10 (2015).

96. Kim, C. H., Park, J. \& Kim, M. Gut microbiota-derived short-chain Fatty acids, T cells, and inflammation. Immune Netw. 14, 277-288 (2014).

97. Preshev, D. \& Van den Ende, W. Fructans: Prebiotics and immunomodulators. J. Funct. Foods 8, 348-357 (2014).

98. Gibson, G. R. \& Roberfroid, M. B. Dietary modulation of the human colonic microbiota: introducing the concept of prebiotics. J. Nutr. 125, 1401-1412 (1995)

99. Pregliasco, F. et al. A new chance of preventing winter diseases by the administration of synbiotic formulations. J. Clin. Gastroenterol. 42, S224-S233 (2008).

\section{ACKNOWLEDGEMENTS}

This work was supported by a MEXT Grant-in-Aid for Scientific Research in Innovative Areas, Japan, "Living in Space", (JP15H05945 to A.T., JP15H05935 to A.T., JP15K21745 to A.T., $18 \mathrm{H} 04971$ to E.H., $18 \mathrm{H} 04968$ to A.K., $16 \mathrm{H} 01631$ to T.A., $18 \mathrm{H} 04989$ to T.A. $16 \mathrm{H} 01638$ to Y.M., $18 \mathrm{H} 04973$ to Y.M., and JP15H05943 to S.F., JP15H05935 to S.F.), and the Institute of Space and Astronautical Science, JAXA Front Loading Study (A.T.) We thank Dr Owen Proudfoot from Edanz Group (www.edanzediting.com/ac) for editing a draft of this manuscript.

\section{AUTHOR CONTRIBUTIONS}

T.A., E.H., M.H., A.K., Y.M., A.T., S.F. designed this study. T.A., K.H., E.H., M.H., A.K., Y.M., A T., S.F. wrote the manuscript.

\section{COMPETING INTERESTS}

The authors declare no competing interests.

\section{ADDITIONAL INFORMATION}

Correspondence and requests for materials should be addressed to T.A.

Reprints and permission information is available at http://www.nature.com/ reprints

Publisher's note Springer Nature remains neutral with regard to jurisdictional claims in published maps and institutional affiliations.

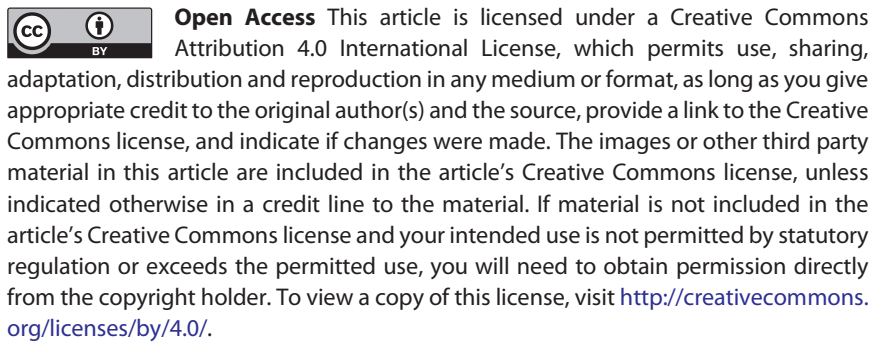

(c) The Author(s) 2020 